\title{
Belphégor
}

\section{Fantômas ou le mythe de "l'homme moderne " chez les poètes des années 1910 et 1920}

\section{Nadja Cohen}

\section{Q OpenEdition}

1 Journals

\section{Édition électronique}

URL : https://journals.openedition.org/belphegor/126

DOI : 10.4000/belphegor.126

ISSN : 1499-7185

Éditeur

LPCM

\section{Référence électronique}

Nadja Cohen, « Fantômas ou le mythe de « l'homme moderne » chez les poètes des années 1910 et 1920 », Belphégor [En ligne], 11-1 | 2013, mis en ligne le 07 juillet 2013, consulté le 07 décembre 2022 URL : http://journals.openedition.org/belphegor/126 ; DOI : https://doi.org/10.4000/belphegor.126

Ce document a été généré automatiquement le 29 septembre 2020.

\section{(c)}

Creative Commons - Attribution - Pas d'Utilisation Commerciale - Pas de Modification 4.0 International - CC BY-NC-ND 4.0

https://creativecommons.org/licenses/by-nc-nd/4.0/ 


\title{
Fantômas ou le mythe de « l'homme moderne » chez les poètes des années 1910 et 1920
}

\author{
Nadja Cohen
}

1 Héros de la série romanesque de Souvestre et Allain, Fantômas fut bien plus qu'une créature de papier. Parler de "mythe» ne semble pas excessif pour qualifier ce ténébreux personnage, dont le nom circule curieusement dans les revues poétiques des années 1910 et 1920. Les très littéraires Soirées de Paris, dont plusieurs contributeurs sont d'ailleurs membres de la mystérieuse "société des amis de Fantômas », ouvrent même un de leurs numéros par la proclamation de la « dictature » du génie du crime et consacrent plusieurs articles à ce personnage. Comment comprendre un tel engouement?

2 À partir de textes d'Apollinaire, de Max Jacob, de Cendrars, d'Aragon et de Desnos, nous montrerons que l'importance conférée au personnage de Fantômas s'explique par le fait qu'il incarne, pour toute une génération, «l'homme moderne », dandy d'un genre nouveau, impassible et amoral, maîtrisant à merveille les technologies et les médias de son temps. Sa devise, «je suis partout », en fait le héros d'une époque fascinée par la " simultanéité » qu'autorisent les nouveaux moyens de communication. Il n'y a donc pas lieu de s'étonner que ce héros de roman soit devenu, dès sa naissance, un personnage de celluloïd, dans le serial en cinq épisodes réalisé entre 1913 et 1914 par Louis Feuillade. Après avoir montré que Fantômas remplit à merveille les caractéristiques de l'homme moderne, dont les poètes précités trouvent le parangon dans le héros de cinéma, nous verrons que ce personnage témoigne également de la grande confusion des valeurs qui agite la génération d'Aragon, ce qui achève de faire de lui une figure-clé dans l'imaginaire collectif de cette période. 


\section{Fantômas, parfait dandy « $1900 »$}

3 Dans un important article intitulé «Du sujet », Aragon définit de la façon suivante le personnage de cinéma :

Les qualités requises du sujet écartent de l'écran tous les prototypes faibles, hésitants, qui discutent sans cesse pour ne jamais agir et qui, par suite, ne pourraient passer à aucun degré pour cinématographiques. On remarquera que ces personnages qui encombrèrent la littérature du siècle dernier se tiennent à l'opposé de l'idéal de l'homme moderne, essentiellement actif et décidé. L'homme moderne seul est un héros de cinéma [...] La vie active qu'il mène le met en état de surexcitation perpétuelle. Il ignore la mélancolie. (Aragon, $1998: 42$.)

4 Même si le poète ne désigne pas ici nommément Fantômas, ce dernier répond parfaitement à une telle définition. Nul n'est moins " hésitant » ou "mélancolique » que le maître de l'angoisse qui fascina la génération d'Apollinaire. Personnage essentiellement actif et parfaitement immoral, Fantômas sacrifie, sans ciller, femmes et enfants sur l'autel de son plaisir. Rappelons qu'il envisage même de faire guillotiner à sa place l'acteur Valgrand qui avait eu l'audace de jouer son rôle au théatre, pour le plus grand plaisir du public. Retournant la ruse du comédien contre son auteur, Fantômas tâche de lui faire endosser pour de bon le rôle du condamné à mort (et y parvient dans le roman de Souvestre et Allain). Dans le film de Feuillade, À l'ombre de la guillotine [1913], seule la vigilance de Juve sauve la vie de l'innocent et relance la traque du héros.

Capable de s'introduire en tous lieux à la faveur de la nuit, de déguisements impeccables ou de la complicité d'agents dormants, Fantômas est partout à la fois. Comme le font remarquer Philippe Azoury et Jean-Marc Lalanne, « Fantômas apparaît lorsque le monde change de vitesse. [...] Fantômas est contemporain de l'essor considérable des médias dont il a aussitôt cherché à faire la plus maitrisable des armes » (Azoury-Lalanne, 92). De fait, les films de Feuillade font la part belle aux moyens de communication modernes : les automobiles, le métro ${ }^{1}$ (qui n'a alors que dix ans), mais aussi le téléphone, les télégrammes (dont l'insertion dans la diégèse remplace habilement celle de cartons explicatifs) et la presse qui relate quasiinstantanément les méfaits du héros. Au forfait de Fantômas succède immédiatement, dans le plan suivant, son récit à la une d'un quotidien parisien, ce qui fait dire aux auteurs du petit ouvrage que « le temps de Fantômas est celui du direct permanent [...] cette "créature de l'ombre et de la nuit, des toits et des égouts [étant] aussi paradoxalement celle des pleins feux des gazettes et de l'éternel grand jour médiatique » (Azoury-Lalanne: 92). Le style de Fantômas même rappelle celui de la publicité : ses cartes de visite à l'encre sympathique laissent sur les lieux du crime son nom «Fantômas", répété à l'envi dans les romans, les films et les affiches de promotion. Dans un chapitre habilement intitulé "Fantômass-média», Azoury et Lalanne saluent la densité du signifiant «Fantômas », qui devient une véritable marque déposée pour le célèbre malfaiteur :

trois syllabes [...] merveilleusement tassées, ramenant à elles l'ensemble de la marque : Fantôme et Masque, Fantôme + Masque = Fantômas $!$ Le concept est d'une efficacité indubitable. [...] Fantômas est un homme du XX $\mathrm{X}^{\mathrm{e}}$ siècle, il s'affiche. [...] Fantômas est à ce point une marque qu'au fil des épisodes, n'importe quelle association de malfaiteurs peut sous-traiter son logo. (Azoury-Lalanne : 91) 
La célèbre affiche du film s'inspire d'ailleurs d'un croquis publicitaire montrant à l'origine un homme géant répandant sur Paris les célèbres "pilules Pink pour personnes pâles ». Choisie par Marcel Allain, cette image fut modifiée pour devenir celle du premier film de la série : À l'ombre de la guillotine ${ }^{2}$. Le cornet de pastilles que tenait le géant a été coupé et remplacé par un poignard ensanglanté (effacé de l'affiche du film), substitution qui fait du bienfaiteur de la ville son plus terrible prédateur. D'une réclame pour le produit miracle, l'affiche anonyme finit ainsi par célébrer Fantômas sur les murs des immeubles de la ville.

\section{Affiche de lancement de la série des Fantômas}

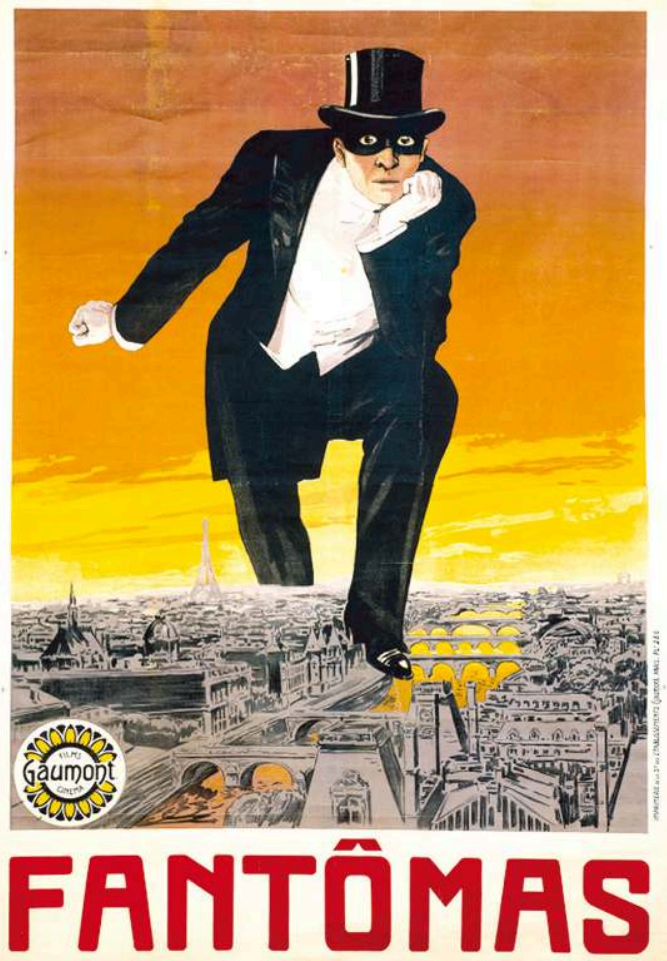

Figure - Affiche de lancement de la série des Fantômas, 1913, lithographie en couleurs, 220x150cm Collection du musée Gaumont.

7 Placardées sur les murs, ces affiches rendent omniprésents les héros de cinéma qui possèdent donc ce « lyrisme de l'ubiquité » dont rêvent les poètes modernistes. «Je suis partout " est aussi bien la devise de la TSF que celle de Fantômas! Pour toutes ces raisons, Fantômas est le parfait héros moderne. Dans Le Mort qui tue ${ }^{3}$, il se joue de la police scientifique aux méthodes balbutiantes en se fabriquant des gants avec de la peau humaine scalpée sur une de ses victimes (tuée à cette seule fin), afin de laisser sur les lieux d'autres crimes les empreintes d'un mort ${ }^{4}$. Le cinéma, qui s'est emparé des épisodes les plus marquants de la saga, contribue au succès des romans, comme l'explique Apollinaire :

Cet extraordinaire roman, plein de vie et d'imagination, écrit n'importe comment mais avec beaucoup de pittoresque, a trouvé, grâce à la vogue que lui a conférée le cinéma, un public cultivé qui se passionne pour les aventures du policier Juve, du journaliste Fandor, de lady Beltham etc. (Apollinaire : 215) 
Il qualifie cette œuvre, ainsi que d'autres romans populaires d'« éloge[s] de l'énergie ». On ne saurait trouver meilleure formule pour résumer la célébration du cinéma par les poètes. Dans un galimatias pseudo-philosophique, Max Jacob célèbre Fantômas pour les mêmes raisons :

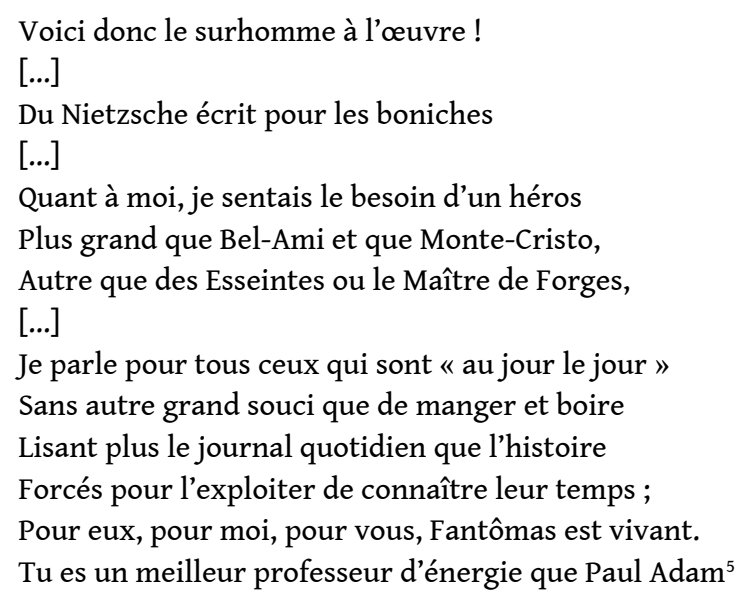

Situé dans la "partie philosophique et morale» de son Écrit pour la S.A.F $F^{6}$ ce texte jongle de façon désinvolte avec des références en vogue à son époque : le « surhomme » de Nietzsche et les écrits de Paul Adam côtoient plaisamment des personnages fictifs : Rodomont, Ubu, Robert Macaire, Bel Ami, Monte Cristo, Des Esseintes et le Maître de forges ${ }^{7}$. Ces références foisonnantes méritent quelques élucidations et en particulier l'expression "professeur d'énergie » que Max Jacob emprunte à Maurice Barrès. Elle apparaît d'abord dans un article du Journal daté du 14 avril 1893, où l'auteur, qui pense que « rien ne manque plus, à cette heure, qu'un peu de volonté dans la race française ", propose comme "psychothérapie» de raconter aux enfants la vie de Bonaparte. L'expression passe surtout à la postérité grâce au roman Les Déracinés (1897), premier volet du cycle du « roman de l'énergie nationale». Plus insolites sont les références à Rodomont, personnage du Roland furieux de l'Arioste, connu pour son insolence et ses fanfaronnades, et à Macaire, personnage d'affairiste sans scrupule de L'Auberge des Adrets, drame représenté pour la première fois en 1823. La disparate de cette énumération a de quoi surprendre. La notion d'énergie "par-delà le bien et le mal » semble en être le dénominateur commun. L'immoralisme et le dandysme en sont d'autres traits caractéristiques. Toutefois, précise Max Jacob, la jeunesse moderne recherche un héros " autre que des Esseintes », ce qui est une façon de donner congé au dandy baudelairien, mélancolique et inactif.

Rappelons que pour Aragon aussi, le héros de cinéma «ignore la mélancolie » (Aragon, 1998, 42). Il semblerait qu'il s'agisse là d'un trait discriminant de l'homme moderne. Contrairement au dandy du XIX ${ }^{\mathrm{e}}$ siècle, "superbe, sans chaleur et plein de mélancolie " ${ }^{8}$ (Baudelaire 1976 (b) : 712), le dandy moderne dont Fantômas est une incarnation, préfère la provocation, le défi lancé à la face du monde, «le plaisir aristocratique de déplaire $»^{9}$ (Baudelaire $1975: 661$ ), qui en est un autre. Au Des Esseintes passif, maladif et passéiste d'À Rebours, se substitue l'image d'un dandy actif comme Jacques Vaché, qu'André Breton qualifie de véritable « Des Esseintes de l'action » (Breton 1973 : 34, nous soulignons).

11 Dans le poème de Max Jacob, Fantômas «propose un idéal aux brigands ", à ceux qui vivent «au jour le jour » car, comme eux, il est «forcé pour l'exploiter de connaître [son] temps». En termes baudelairiens, il est riche d'une " intelligence subtile de tout 
le mécanisme moral de ce monde» (Baudelaire 1976 (a) : 957, cité par Breton 1992: $957)^{10}$ et rejette les modèles que la société lui propose, afin de définir un « héroïsme de la vie moderne $»^{11}$, dans lequel l'humour noir tient une place prépondérante.

Ainsi pourrait s'expliquer l'incroyable vogue suscitée par Fantômas et dont témoigne de façon exemplaire la fondation de la S.A.F, "Société des Amis de Fantômas ", pour laquelle Max Jacob écrivit ce texte. Selon André Salmon, «c'est Guillaume Apollinaire qui eut l'idée maîtresse d'une société des amis de Fantômas, dix ans avant le Surréalisme »(Salmon : 234). Peu structurée, cette société apparaissait

à la fois aussi irréelle, aussi imaginaire que le fameux Stendhal-Club. [...] Ni statuts, ni local, ni but défini, pas de bureau. Mais partout où les membres, triés sur le volet, se rencontraient, il y avait nécessairement, et comme de fait, tenue de S.A.F. (Salmon : 234)

Pour sa part, Salmon refuse d'en faire partie, ne pouvant « rien accorder à de telles œuvres, non méprisables certes, mais absolument dépourvues d'intention artistique " (Salmon : 233). Max Jacob, en revanche, "y adhéra d'enthousiasme » et «fut quelque chose comme le prieur, le chapelain, le directeur d'inconscience des Amis de Fantômas" (Salmon : 234). Sous le charme du brigand, la S.A.F se piquait parfois de passer elle-même à l'action. André Salmon rapporte que « ceux de la S.A.F ornèrent de mystérieux graffiti les murs principaux du vieux Montmartre; les murs croulants de la Butte, couronnés en juin de lilas enivrants. De bons bourgeois s'émurent de certaine Main sanglante. Devait-on craindre - en rouge - ce qu'ailleurs fut la Mano Negra? $»^{12}$ (Salmon: 234). Il faut ajouter que, peut-être dans un souci de réalisme, les poètes avaient recruté dans le milieu quelques bandits, "deux ou trois antiques voyous, figurants apaisés du Lapin Agile, alias "Cabaret des Assassins ", et qu'on flattait en les tenant, sur parole, pour des assassins tout de bon, allaient tenir emploi d'Amis actifs quand des écrivains notoires n'obtenaient que l'honorariat » (Salmon : 233). Ces plaisanteries de potaches ont aussi donné naissance à des poèmes, voire à des rivalités entre poètes. Dans le numéro 26 de juin 1914 des Soirées de Paris, Cendrars célébrait son voyage littéraire dans les aventures de Fantômas. Fâché d'avoir été devancé, Max Jacob se fend d'un Écrit pour la SAF, le mois suivant, comme en témoigne Apollinaire dans une lettre à Picasso :

«Max lit toujours Fantômas ainsi que moi et tout le monde mais, furieux que Cendrars ait déjà fait un poème sur Fantômas, il en prépare un autre destiné à rivaliser avec le premier $»^{13}$.

Le long texte de Max Jacob, dédié à madame Picabia, est divisé en quatre sections : une célébration du bandit, une "partie critique", analysant le schéma classique d'un volume des aventures de Fantômas, une critique acérée du style des auteurs et enfin une partie philosophique et morale, citée ci-dessus, où Max Jacob énumère les raisons de son admiration. C'est en fait tout le numéro 27 des Soirées de Paris qui est placé sous le signe de la " dictature de Fantômas » :

Une dictature aux Soirées de Paris ${ }^{14}$

Profitant de l'absence des rédacteurs (ils sont à Châtillon pour acheter une chienne de garde), je constitue ma dictature.

Article unique : les collaborateurs des Soirées de Paris sont instamment priés de ne plus envahir la revue avec leurs visions de Fantômas, car élever un roman policier à la hauteur d'une épopée mondiale, c'est encore encourager l'autorité à prendre les libertés les plus imprévues. signé : Fantômas. 
Dans ce même numéro, Maurice Raynal, chargé de la chronique cinématographique de la revue, manifeste également son enthousiasme sans borne pour le "génie du crime ». $\mathrm{Au}$ terme de sa chronique, il fait l'apologie du film de Feuillade :

Et maintenant, oserai-je... n'oserai-je pas ? Enfin, courage, et à la grâce de Dieu maintenant... Fantômas.

O noblesse ! ô beauté! [...] dans ce film, touffu, compact et concentré, que de génie éclate! Tenez, la cheminée à gaz, par exemple. Un marquis, souffrant, se couche après avoir fait allumer sa cheminée à gaz. Que fait alors Fantômas? Il court au compteur, le ferme, et la flamme s'éteint. Ce n'est pas tout. En effet, très posément, il ouvre à nouveau le compteur: le gaz se répand dès lors dans la pièce, et cet aristocrate est asphyxié! Comme c'est simple, comme c'est grand ! [...] Et, plus loin, voyez avec quelle délicatesse le héros «emprunte » 500.000 francs à la marquise. Comme vous dites, en effet, M. Fantômas car je n'ose écrire Fantômas tout court, comme vous dites en effet, monsieur, pour vous excuser: "Fantômas a faim quelquefois! » C'est du meilleur Hugo, et c'est trop beau, vraiment! Pour moi, je pense que Fantômas est un roman à clef; mais qui nous la donnera ? Enfin, qui vivra verra. Cependant, des personnes bien informées me disent que Fantômas serait le frère de Juve le policier. Serait-ce possible, mon Dieu? Moi qui aurait juré qu'ils ne faisaient qu'un seul et même personnage !15

Que ce tourbillon énergétique s'exerce dans un but louable ou non est indifférent. C'est même clairement l'amoralité, voire l'immoralité des personnages qui a la faveur du public. L'énumération fascinée des méfaits de Fantômas dans le texte de Raynal témoigne de cette dépense d'énergie et de l'ingéniosité de ce technicien du crime. La vivacité du texte, "touffu, compact et concentré », mime celle du film : le présent de narration, les phrases brèves, l'abondance des verbes d'action confèrent à l'article un rythme alerte. Le récit est interrompu de loin en loin par les exclamations enthousiastes du chroniqueur devant la beauté des crimes commis, le plus souvent, aux dépens de l'aristocratie. On peut s'interroger sur cet éloge du crime qu'on trouve sous la plume de bien des auteurs de l'époque. Faut-il y voir une simple provocation, un rappel du principe - bien connu - selon lequel on ne fait pas de littérature avec des bons sentiments, ou plus profondément un fait de génération?

\section{Fantômas ou le « soleil » du crime »}

La fascination pour la violence et l'audace du hors-la-loi n'est certes pas un fait nouveau. Comme l'écrit Francis Lacassin :

Par leur contenu latent, les romans de Souvestre et Allain répondaient bien à l'attente d'un inconscient collectif : leur ténébreux héros assumait les aspirations et les désirs inexprimés que chacun pouvait ainsi assouvir sans danger par la lecture. [...] Fantômas répondait encore à un insatiable appétit de merveilleux et de violence. (Lacassin : 97)

En plaçant Fantômas dans la lignée des « contes de fées, purs produits de l'imagination collective, où l'érotisme et la violence se donnent libre cours ", l'essayiste souligne la dimension intemporelle d'un tel phénomène, «la cruauté et le goût de la violence sommeillant chez tout homme ». Mais Aragon juge plus intéressant d'étudier les formes que prend cette violence à des moments historiques donnés. C'est ce qu'il suggère quand, soucieux d'écrire la mythologie de sa génération, il écrit que « les films policiers [sont] aussi significatifs de cette époque que se montrèrent de leurs siècles les romans de chevalerie, les romans précieux ou les romans libertins » (Aragon, 1998 : 39). En cela 
il rencontre André Breton qui se demande également en quoi Fantômas participe à «l'élaboration du mythe collectif propre à [une] époque " (Breton 1999: 667), en en révélant le « contenu latent » et non le contenu manifeste ${ }^{16}$.

L'exaltation de personnages marginaux est déjà un phénomène massif, voire un poncif, pendant la période romantique. Le brigand, type Hernani, devient même la figure héroïque par excellence. Mais, le moins que l'on puisse dire est que, pour être révolté, Hernani est tout sauf une figure du mal radical. Fantômas s'inscrit dans une tout autre lignée. Si Fantômas date d'avant la guerre, les lectures qui en sont faites a posteriori nous semblent révélatrices du cataclysme idéologique de la Première Guerre mondiale. Pour le poète roumain Benjamin Fondane, sympathisant dada, l'engouement de la jeunesse pour les films d'action est une conséquence d'un nouveau mal du siècle :

À d'aucuns, cette conception de la vie peut sembler puérile, mais nous savons combien nous avions besoin qu'on exaltât, qu'on stimulât, qu'on fouettât notre jeunesse fatiguée de vieux conquistadors de l'ennui. (Fondane : 69)

Cette « fatigue » est associée par Fondane à un climat de renversement des valeurs,

Depuis que l'élite nous impose les valeurs des faibles, des valeurs de faillite : vertu, résignation, exaltation du travail, bonheur bon marché, c'est chez la populace et plus bas chez la pègre, chez la basse pègre que se sont réfugiées les valeurs aristocratiques: le courage, le goût du risque, l'appel du danger, le mépris de la mort. Vous n'êtes pas sans avoir remarqué que ce ne sont point aujourd'hui les rois, ni les fils de rois ni les castes nobles qui alimentent notre faim terrible, notre fringale de héros. L'admiration des foules se porte, canalisée par le journal, vers les bandits des cours d'assises, vers les forçats évadés, vers les explorateurs des terres vierges, vers les aviateurs qui se tuent à vingt ans [...]. (Fondane : 66-67) ${ }^{17}$

Dans une perspective sociologique, Aragon lie, plus clairement encore, cette célébration provocatrice des bandits au climat de déroute idéologique de la Première Guerre. Dans Pour expliquer ce que j'étais, il fera plus tard état du climat des premières années de guerre et de la façon dont cette dernière était perçue par la jeunesse :

J'étais de ceux qui sachant qu'ils allaient partir à leur tour et ne croyant pas à l'avenir, à la vie qui continue, entendaient s'en payer leur saoul de jeunesse, et vomissaient les homélies patriotiques et le bourrage de crâne, les poncifs de la guerre, la tartufferie de ces gens d'âge et de raison qu'ils voyaient à l'arrière s'envoyant les femmes jeunes de ces jeunes hommes absents. [...] Parce que cette guerre-là, on voyait trop de quoi elle était faite. C'était une guerre des vieux, pour des raisons qui avaient exalté les vieux, qui ne touchaient pas les jeunes, et c'étaient les jeunes qui la faisaient pour les vieux. (Aragon 1989: 35)

Ensevelie dans la boue des tranchées, la foi de la jeunesse cède la place à une fatigue mentale (celle des « vieux conquistadors de l'ennui » dont parle Fondane), à un certain relativisme et à la recherche de satisfactions immédiates. La jeune génération cherche ailleurs que dans le patriotisme de ses pères un motif d'exaltation :

À quelle lumière voyions-nous, qui n'avions pas vingt ans, la vie bouleversée, nous autres au début de la guerre, sur le seuil des écoles? [...] Tout ce qui touchait proprement à la guerre, [...], cet exhibitionnisme de l'horreur, nous répugnait si fortement que je ne crois pas mentir en disant que jamais la guerre ne fut loin [sic] du cœur des jeunes gens qu'en ces jours qu'elle dominait les adultes. [...]

À ce point étonnant de confusion morale, [...], comment [les] jeunes ne se fussent-ils point reconnus dans ces bandits splendides, leur idéal et leur justification? Et pour rendre plus exaltante cette constatation, cet enthousiasme défendu, les journaux dénonçaient le cinéma, école du crime. Les enfants en proie à la révision des idées n'allaient-ils pas se parer à leurs propres yeux d'un prestige incomparable en 
acceptant secrètement ce lieu commun de la presse ? Oui, ils couraient où les appelait le CRIME, le seul soleil qui ne fut point encore sali. (Aragon 1994: 8)

\section{de premier ordre. Fan}

Si Fantômas est politique, c'est alors, peut-être, de la même façon qu'il est dandy : politicien sans programme (et sans morale) autant que rebelle sans cause. Agissant par simple soif d'assouvir des désirs régulièrement appelés à être réveillés. Le scandale permanent qu'est l'existence de Fantômas, le chaos social qu'il invite à suivre, le désordre que laisse son passage, en ont vite fait [...] un anar [sic] virulent [...]. Fantômas peut en effet se vanter de quelques actions directes dans un Paris qui dort et qui lui appartient, bafouant les institutions (la police en premier lieu) ou ses monuments symboliques. Ceci aidé par sa quasi-contemporanéité avec les méfaits de la bande à Bonnot, qu'il devance de six mois. (Azoury-Lalanne : 24)

Lacassin rappelle d'ailleurs que «la guerre avec son cortège de préoccupations nouvelles [...] devait effacer la Belle Epoque et suspendre les exploits du criminel magnifique " pour plusieurs raisons et notamment parce qu'on reprochait au film « de faire l'apologie d'un criminel et même d'avoir inspiré et préparé les méfaits de Bonnot» (Lacassin: 100). Pour sa part, il juge pourtant inadéquat le terme d' « anarchiste " pour qualifier Fantômas :

Fantômas, ce n'est pas le larron qui plait et qui amuse parce qu'il se joue impunément des gendarmes; ce n'est pas le révolté anarchiste qu'on admire parce qu'il bafoue une police pourtant puissante et se dresse contre une société sophistiquée. C'est aussi le criminel qui, par sa cruauté, son goût de l'or et du meurtre, piétine allègrement l'ordre social et moral. (Lacassin : 97)

Que le message politique de Fantômas soit explicite ou non, le fait qu'il "piétine allègrement l'ordre social et moral» le constitue de facto en agent subversif. Le personnage s'avérant être le frère caché de l'inspecteur Juve, son ennemi de toujours, l'inquiétante proximité entre le crime et la loi rend la série d'autant plus troublant et achève de nous persuader que son succès n'est rien moins qu'anecdotique. Maître en communication, "professeur d'énergie ", agent subversif auréolé du "soleil noir du crime ", dandy affranchi de la morale commune à une époque d'intense révision des valeurs : Fantômas est tout cela à la fois. En cela il incarne une des formes de l'homme moderne. On ne saurait donc s'étonner si, de tous les feuilletons populaires aimés par 
les poètes, celui-ci laissa une empreinte aussi profonde dans le paysage mental de toute une génération.

\section{BIBLIOGRAPHIE}

Apollinaire, Guillaume, « Fantômas », La Vie anecdotique, 16 juillet 1914, repris dans CEuvres en prose complètes III, édition de Pierre Caizergues et Michel Décaudin, 1993.

Aragon, Louis, « Du sujet ", Le Film, 6è année, nouvelle série, n 149, 22 janvier 1919, repris dans Chroniques : 1918-1932, édition de Bernard Leuilliot, Stock, 1998.

Aragon, Louis, Pour expliquer ce que j'étais [1942-1943], Gallimard, NRF, 1989.

Aragon, Louis, «Les Vampires » [1923], Projet d'histoire littéraire contemporaine, éd. Marc Dachy, Gallimard-Digraphe, 1994.

Azoury, Philippe et Lalanne, Jean-Marc, Fantômas style moderne, Centre Pompidou-Yellow now, 2002.

Baudelaire, Charles, Fusées, XII, Euvres Complètes I, édition établie par Claude Pichois, Gallimard, (Bibliothèque de la Pléiade), 1975.

Baudelaire, Charles, Le Peintre de la Vie Moderne, III : « L'artiste, homme du monde, homme des foules et enfant ", Salon de 1859, CEuvres complètes II, texte établi, présenté et annoté par Claude Pichois. Gallimard, (Bibliothèque de la Pléiade), 1976 (a).

Baudelaire, Charles, le Peintre de la Vie Moderne, IX, « le dandy ", Salon de 1859, CEuvres complètes II, texte établi, présenté et annoté par Claude Pichois. Gallimard, (Bibliothèque de la Pléiade), 1976 (b).

Breton, André, deuxième entretien, Entretiens, écrits et enregistrés en 1951, radiodiffusés de février à juin 1952. Gallimard, 1969, (Idées),

Breton, André, l'Anthologie de l'Humour Noir, Euvres complètes II, édition établie par Marguerite Bonnet, avec, pour ce volume, la collaboration de Philippe Bernier, Étienne-Alain Hubert et José Pierre. Gallimard, (Bibliothèque de la Pléiade), 1992.

Breton, André, «Limites non-frontières du surréalisme », La Clé des Champs, CEuvres complètes III, édition établie par Marguerite Bonnet, avec, pour ce volume, la collaboration de Philippe Bernier, Marie-Claire Dumas, Étienne-Alain Hubert, José Pierre. Gallimard, (Bibliothèque de la Pléiade), 1999.

Fondane, Benjamin, « Présentation de films purs » [1929], Écrits pour le cinéma : le muet et le parlant [Plasma, 1984], textes réunis et présentés par Michel Carassou, Olivier Salazar-Ferrer et Ramona Fotiade, Verdier (Poche), 2007.

Lacassin, Francis, Pour une contre-histoire du cinéma, Union générale d'éditions (10-18), 1972.

Picasso-Apollinaire, Correspondance, éd. Par Pierre Caizergues et Hélène Seckel, GallimardRéunion des musées nationaux, 1992.

Salmon, André, Souvenirs sans fin, tome II, Gallimard, 1961. 


\section{NOTES}

1. Emprunté par la prostituée dans le deuxième épisode de la série, Juve contre Fantômas.

2. Dans un état intermédiaire, l'affiche, qui fut placardée sur tous les murs de Paris pour lancer la série des volumes début 1911, montrait Fantômas tenant en main un poignard ensanglanté qui fut supprimé pour l'affiche du film.

3. Louis Feuillade, Le Mort qui tue [1913], troisième épisode de Fantômas.

4. Notons que cet épisode terrifia une jeune spectatrice devenue célèbre depuis: Nathalie Sarraute évoque, dans Enfance, son effroi enfantin face à ce cruel stratagème de Fantômas.

5. Max Jacob, «Écrit pour la S.A.F », Les Soirées de Paris, n²6-27, juillet-août 1914. [Rappelons qu'il s'agit du numéro des Soirées de Paris dans lequel est décrétée la dictature du bandit masqué.] 6. La « S.A.F » est la Société des Amis de Fantômas.

7. Cuvre de Georges Ohnet publiée en 1882.

8. « Le dandysme est un soleil couchant; comme l'astre qui décline, il est superbe, sans chaleur et plein de mélancolie » (Baudelaire 1976 (a) : 712).

9. «Ce qu'il y a d'enivrant dans le mauvais goût, c'est le plaisir aristocratique de déplaire » (Baudelaire 1975 : 661, cité par Breton 1992 : 957).

10. Sans doute pour étayer son propre point de vue, Breton ne donne pas la phrase complète de Baudelaire que voici : «Le mot dandy implique une quintessence de caractère et une intelligence subtile de tout le mécanisme moral de ce monde; mais d'un autre côté, le dandy aspire à l'insensibilité [...], le dandy est blasé, ou il feint de l'être, par politique et raison de caste ».

11. Selon l'expression utilisée par Baudelaire dans le Salon de 1846, XVIII (Baudelaire 1976 : 493).

12. La Mano Negra passait à l'époque pour une organisation anarchiste, secrète et violente, ayant agi en Andalousie à la fin du XIX siècle et à laquelle on attribuait des assassinats ainsi que des incendies de récoltes ou de bâtiments. Les historiens tendent maintenant à y voir des révoltes de la faim, qualifiées d' "anarchistes » par un gouvernement désireux de les discréditer et de les mater.

13. Lettre d'Apollinaire à Picasso, datée du 4 juillet 1914 (Picasso-Apollinaire : 119).

14. « Nous ne sommes pour rien dans la rédaction de la présente note. Nous ne nous sommes aperçus de son insertion que le numéro imprimé. Il était trop tard pour la faire sauter. À peine avons-nous pu insérer en bas de page cette faible protestation. On notera plus loin, à propos du poème de M. Max Jacob, la constitution d'une S.A.F (Société des Amis de Fantômas) qui ne nous paraît ni moins mystérieuse ni moins digne d'intérêt que le Stendhal-Club ou le Baudelaire-Club. Les rédacteurs des Soirées de Paris. "

15. Les Soirées de Paris, $\mathrm{n}^{\circ} 27$, juillet-août 1914.

16. En vertu de ce principe, Breton fait du «fantastique » la clé permettant de «toucher ce fond historique secret qui disparaît derrière la trame des événements». Dans cette optique, Breton considère «le genre noir » comme «pathognomonique du grand trouble social qui s'empare de l'Europe à la fin du XVIII ${ }^{\mathrm{e}}$ siècle ». Sade, déjà, dans son Idée sur les romans, en faisait «le fruit indispensable des secousses révolutionnaires dont l'Europe entière se ressentait " et cette secousse sera sensible jusqu'au XIX ${ }^{\mathrm{e}}$ siècle, dont le fameux goût pour les ruines. Ce dernier est analysé comme suit par Breton: «Les ruines n'apparaissent brusquement si chargées de signification que dans la mesure où elles expriment visuellement l'écroulement de la période féodale; le fantôme inévitable qui les hante marque, avec une intensité particulière, l'appréhension du retour des puissances du passé ; les souterrains figurent le cheminement lent, périlleux et obscur de l'individu humain vers le jour ; la nuit orageuse ne transpose que le bruit, à peine intermittent du canon.» (Breton 1999: 666) Comme souvent, Breton superpose ici deux grilles de lecture: le symbolisme historique, d'une part, et, d'autre part, la psychanalyse, à laquelle il emprunte la notion de retour du refoulé, qui devient sous sa plume «l'appréhension du retour des puissances du passé ». 
17. On pourrait voir la même tentation à l'œuvre dans les Vies imaginaires de Marcel Schwob qui y narre celles de pirates, de meurtriers (Burke \& Hare).

18. Max Jacob, Ecrit pour la S.A.F, op.cit.

\section{RÉSUMÉS}

À partir d'un choix de textes d'Apollinaire, de Max Jacob, de Cendrars, d'Aragon et de Robert Desnos, cet article explique l'importance conférée au personnage de Fantômas par le fait qu'il incarne pour cette génération "l'homme moderne ", dandy d'un genre nouveau, impassible et amoral, maîtrisant à merveille les technologies et les médias de son temps.

\section{INDEX}

Mots-clés : poésie, cinéma, art, intermédialité, Aragon Louis, Apollinaire Guillaume, Breton André, Desnos Robert, Soupault Philippe, Fondane Benjamin, Cendrars Blaise, Jacob Max, Fantômas

\section{AUTEUR}

\section{NADJA COHEN}

Ancienne élève de l'École normale supérieure (ENS-LSH), agrégée de lettres modernes, Nadja Cohen est titulaire d'une thèse de doctorat, à paraître en septembre 2013 aux Classiques Garnier sous le titre Les poètes modernes et le cinéma (1910-30). Elle a travaillé à l'université Grenoble IIIStendhal en qualité d'AMN puis d'ATER et enseigne actuellement dans le secondaire. Elle est l'auteur d'articles sur Henri Michaux et sur André Breton mais a surtout écrit sur les relations entre le cinéma et les poètes Guillaume Apollinaire, Benjamin Fondane, Louis Aragon, Philippe Soupault et Blaise Cendrars. Elle s'intéresse plus largement aux rapports entre la poésie et les arts, la poésie et les médias, sujet d'un colloque qu'elle a co-organisé en novembre 2008 à la Sorbonne et dont les actes ont paru en 2012 chez Nouveau monde éditions sous le titre Poésie et médias XX-XXIe siècle. 